\title{
Bilateral Primary Mucinous Carcinoma of the Eyelid
}

Christopher K.H. Burris, M.D. ${ }^{1,2}$, Meisha L. Raven, D. O. ${ }^{1,2}$, Maria E. Rodriguez, M.D. ${ }^{1,2}$, Heather D. Potter, M.D. ${ }^{1,2}$, Mark J. Lucarelli, M.D. ${ }^{1,2}$, and Daniel M. Albert, M.D., M.S. ${ }^{1,2}$ ${ }^{1}$ Department of Ophthalmology, The University of Wisconsin, Madison, WI

${ }^{2} \mathrm{McPherson}$ Eye Research Institute, Madison, WI

\begin{abstract}
The aim of this study is to report a case of bilateral primary mucinous carcinoma of the eyelids. This is a case report and literature review. A 71 year-old-female presented with primary mucinous carcinoma of the left upper eyelid, which was excised with Mohs surgery. One year later she developed primary mucinous carcinoma of the right upper eyelid, which was also treated Mohs surgery. Extensive workup was negative for evidence of an unknown primary carcinoma or metastasis. Primary mucinous carcinoma of the eyelids may occur as multifocal tumors, and bilateral disease is not necessarily indicative of metastatic disease.
\end{abstract}

\section{Introduction}

Primary mucinous carcinoma of the skin (PMCS) is a rare adnexal neoplasm originating from eccrine sweat glands. ${ }^{1}$ Approximately $75 \%$ of cases originate in the head and neck, with $40 \%$ of cases in the periorbital region. ${ }^{2}$ PMCS frequently recurs locally, but rarely metastasizes to the lymph nodes (5-15\%) or distant tissues (2-7\%). ${ }^{3,4}$ Before making the diagnosis of PMCS, internal malignancy must be ruled out, as these lesions may be confused with metastatic carcinoma. We present a case of bilateral PMCS. The collection and evaluation of protected patient health information was HIPAA-compliant.

\section{Case Report}

A 71-year-old female underwent excision of a mildly erythematous left upper eyelid cystic mass (Fig. 1A). Histopathology showed a well-circumscribed mass containing lobules of cells within a mucoid matrix (Fig. 2A, B). Rare mitotic figures were seen, and there was focal staining of the myoepithelial cells with the p63 stain (Fig. 3A). She was diagnosed with mucinous carcinoma and underwent slow Mohs surgery with delayed reconstruction after histopathologic analysis of paraffin fixed tissue (Fig. 1C). Due to concern that this could represent metastatic disease, the patient underwent a colonoscopy, mammogram, and computed tomography (CT) of the face/sinus, neck, chest, abdomen, and pelvis, which were

Correspondence: Daniel M. Albert, MD, MS, Department of Ophthalmology and Visual Sciences, University of Wisconsin, RM. K6/412, Clinical Science Center, 600 Highland Ave., Madison, WI, 53705-3611, USA, dalbert@ wisc.edu, Phone: (608) 262-4800, Fax: (608) 263-0543.

Conflict of Interest: None of the authors have any propriety interests, financial competing interests, or conflicts of interest related to this submission. 
negative for any evidence of tumor. Serum cancer antigens 19-9, 27-29, and 15-3 (CA 19-9, CA 27-29, CA 15-3), and carcinoembryonic antigen (CEA) were all within normal limits. One year later she developed a subtle right upper eyelid mass with madarosis lacking pigmentation, erythema, scale, ulceration, or telangiectatic vessels (Fig. 1B). Incisional biopsy showed features similar to her previous biopsy, and was positive for p63 (not shown), and cytokeratin 7 (CK7), but negative for cytokeratin 20 (CK20) (Fig. 3B, C). Again she underwent slow Mohs assisted by alcian blue staining to highlight the mucin component of the tumor. The tumor proved more extensive than expected from the size of the clinical nodule (Fig. 1B), and required 3 Mohs surgery sessions over 7 days (Fig. 1D). Subsequent eyelid reconstruction required a semicircular myocutaneous Tenzel advancement flap. At her 2 month follow up appointment her vision was 20/20 OU, with the rest of her exam within normal limits.

\section{Discussion}

PMCS is rare, but bilateral disease is even rarer with only one other case reported in the literature to our knowledge. ${ }^{5}$ It should be a diagnosis of exclusion, after an internal malignancy has been ruled out. Clinically this was done with colonoscopy, mammography, CT scan, and serum markers CA 19-9, CA 27-29, CA 15-3, and CEA in this case, but positron emission tomography-computed tomography (PET-CT) and magnetic resonance imaging (MRI) could also play a role. It is important to note that the face is a rare location (6\%) for cutaneous metastasis from internal malignancy, and when mammary and intestinal neoplasms involve the skin, it is most often the skin immediately overlying the lesions. ${ }^{6,7}$

Primary lesions of the skin tend to stain positively for p63, as opposed to mucinous carcinoma metastatic to the skin. ${ }^{8}$ Kazakov et al found positive CK7 and negative CK20 staining to be a consistent feature of primary skin neoplasms. They noted "dirty necrosis", or necrotic eosinophilic foci containing nuclear debris to be common in tumors of intestinal origin. Tumors originating from the breast can have similar cytokeratin staining patterns as well as estrogen and progesterone receptors, making them indistinguishable from skin primaries. ${ }^{9}$

The classic behavior of PMCS involves slow growth with local recurrence, but these indolent tumors maintain the potential for aggressive growth and metastasis. ${ }^{10}$ One case documented six local recurrences without metastasis over 30 years. ${ }^{11}$ The mainstay of treatment is surgical excision, but remission has been achieved with the aromatase inhibitor Anastrozole in a metastatic estrogen receptor positive case. ${ }^{12}$ We recommend complete excision, with slow Mohs (staged excision where margins are processed as permanent specimens as opposed to frozen sections) aided by mucin staining (e.g., PAS, alcian blue, mucicarmine, colloidal iron) or immunohistochemistry. ${ }^{13,14}$ Although there should always be strong suspicion for a distant primary tumor in PMCS, bilateral disease does not necessarily signify metastasis.

\section{Acknowledgments}

Grant Support: Supported in part by an unrestricted grant to the Department of Ophthalmology and Visual Sciences from Research to Prevent Blindness, Inc. NY, NY., NIH core grant P30 EY016665 


\section{References}

1. Headington JT. Primary mucinous carcinoma of skin: histochemistry and electron microscopy. Cancer. 1977 Mar; 39(3):1055-63. [PubMed: 199341]

2. Snow SN, Reizner GT. Mucinous eccrine carcinoma of the eyelid. Cancer. 1992 Oct 15; 70(8): 2099-104. [PubMed: 1327486]

3. Wright JD, Font RL. Mucinous sweat gland adenocarcinoma of eyelid: a clinicopathologic study of 21 cases with histochemical and electron microscopic observations. Cancer. 1979 Nov; 44(5):175768. [PubMed: 227576]

4. Sanke RF. Primary mucinous adenocarcinoma of the eyelid. Ophthalmic Surg. 1989 Sep; 20(9):66871. [PubMed: 2554230]

5. Bertagnoli R, Cook DL, Goldman GD. Bilateral primary mucinous carcinoma of the eyelid treated with Mohs surgery. Dermatol Surg. 1999 Jul; 25(7):566-8. [PubMed: 10469115]

6. Brownstein MH, Helwig EB. Metastatic tumors of the skin. Cancer. 1972 May; 29(5):1298-307. [PubMed: 4336632]

7. Kazakov DV, Suster S, LeBoit PE, Calonje E, Bisceglia M, Kutzner H, Rütten A, Mentzel T, Schaller J, Zelger B, Baltaci M, Leivo I, Rose C, Fukunaga M, Simpson RH, Yang Y, Carlson JA, Cavazza A, Hes O, Mukensnabl P, Vanecek T, Fidalgo A, Pizinger K, Michal M. Mucinous carcinoma of the skin, primary, and secondary: a clinicopathologic study of 63 cases with emphasis on the morphologic spectrum of primary cutaneous forms: homologies with mucinous lesions in the breast. Am J Surg Pathol. 2005 Jun; 29(6):764-82. [PubMed: 15897743]

8. Ivan D, Nash JW, Prieto VG, Calonje E, Lyle S, Diwan AH, Lazar AJ. Use of p63 expression in distinguishing primary and metastatic cutaneous adnexal neoplasms from metastatic adenocarcinoma to skin. J Cutan Pathol. 2007 Jun; 34(6):474-80. [PubMed: 17518775]

9. Breiting L, Dahlstrøm K, Christensen L, Winther JF, Breiting V. Primary mucinous carcinoma of the skin. Am J Dermatopathol. 2007 Dec; 29(6):595-6. [PubMed: 18032965]

10. Miyasaka M, Tanaka R, Hirabayashi K, Yamazaki A, Shinohara H, Taira H, Akamatsu T. Primary mucinous carcinoma of the skin: a case of metastasis after 10 years of disease-free interval. Eur $\mathrm{J}$ Plast Surg. 2009 Aug; 32(4):189-193. [PubMed: 20234869]

11. Burris CK, Rajan KD, Iliff NT. Primary mucinous carcinoma of the periocular region: successful management with local resections over 30 years. BMJ Case Rep. 2013 Feb.15:2013.

12. Shockman S, Krug L, Lountzis N. Remission of metastatic primary mucinous carcinoma of the skin with anastrozole. J Am Acad Dermatol. 2014 Jul; 71(1):e27-8. [PubMed: 24947711]

13. Papalas JA, Proia AD. Primary mucinous carcinoma of the eyelid: a clinicopathologic and immunohistochemical study of 4 cases and an update on recurrence rates. Arch Ophthalmol. 2010 Sep; 128(9):1160-5. [PubMed: 20837800]

14. Marra DE, Schanbacher CF, Torres A. Mohs micrographic surgery of primary cutaneous mucinous carcinoma using immunohistochemistry for margin control. Dermatol Surg. 2004 May; 30(5):799_ 802. [PubMed: 15099329] 


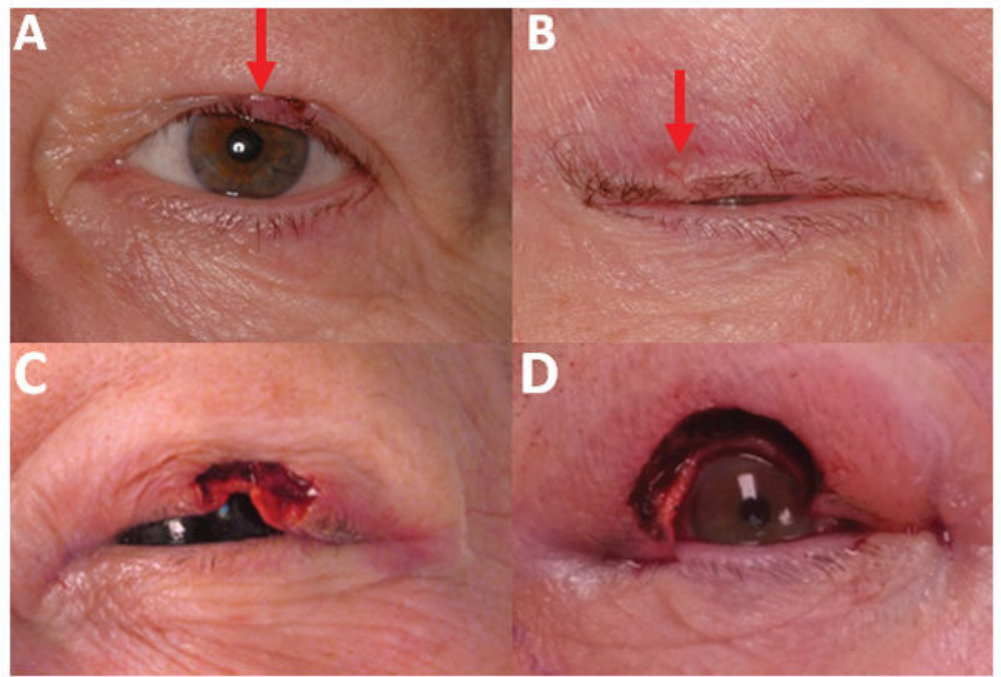

Figure 1.

A. Left eye erythematous mass before Mohs, B. Right eye non-erythematous mass before Mohs. Note mild madarosis (arrows) C. Left eye after Mohs, D. Right eye after Mohs 


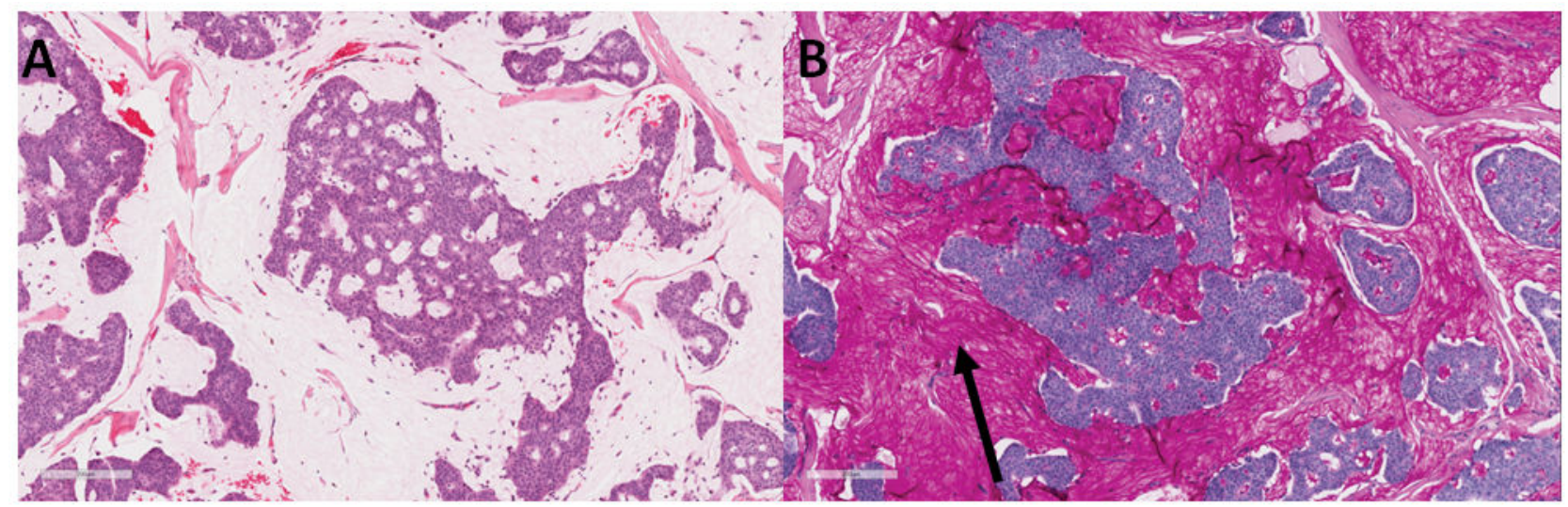

Figure 2.

A. Lobulated pools of mucin, with floating islands of tumor cells and thin trabeculae (Hematoxylin and Eosin, 20x), B. Mucin highlighted by periodic acid-Schiff stain (Arrow, PAS, 20x) 


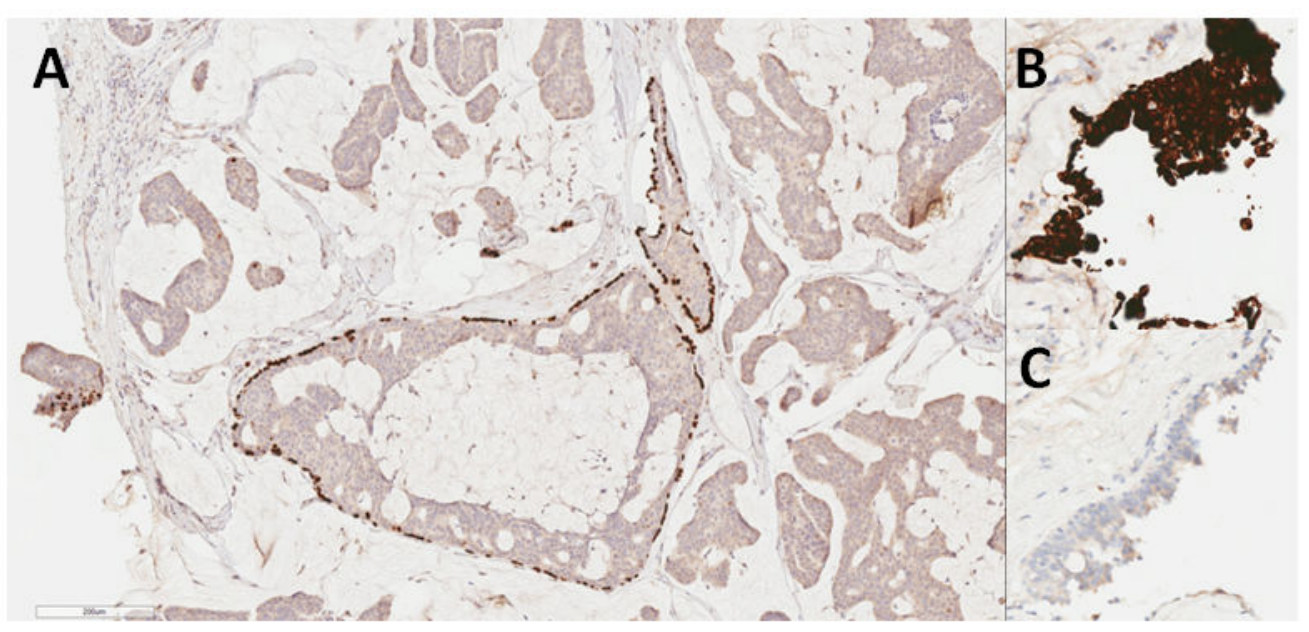

Figure 3.

A. P63 staining in the myoepithelial cell layer (p63, 20x), B. Positive CK7 staining (CK7, 20x), C. Negative CK20 staining (CK20, 20x) 